\title{
Caracterización de bacilos gramnegativos multi-resistentes, aislados en pacientes hospitalizados en instituciones de salud de Barranquilla (Colombia)
}

\author{
Characterization of multiresistant gram-negative bacilli, isolated in patients \\ hospitalized in health institutions in Barranquilla (Colombia)
}

\author{
Marlene Guerra-Sarmiento' ${ }^{1}$ Fernando Ruíz-Martin Leyes' ${ }^{1}$ Laura Arzuza-Ortega ${ }^{1}$ y Ronald Maestre-Serrano ${ }^{2}$
}

\begin{abstract}
'Laboratorio de Salud Pública del Atlántico, Secretaría de Salud del Atlántico, Barranquilla, Colombia.
${ }^{2}$ Universidad Simón Bolívar. Facultad de Ciencias de la Salud.

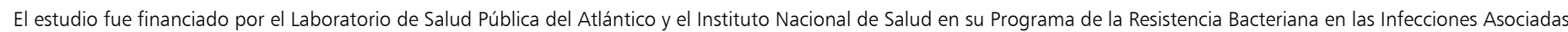
a la Atención en Salud.

Los autores declaran no tener conflictos de interés.
\end{abstract}

Recibido: 5 de febrero de 2020 (segunda versión: 9 de febrero de 2021) / Aceptado: 1 de marzo de 2021

\section{Resumen}

Introducción: La resistencia a carbapenémicos en bacilos gramnegativos es un problema de salud pública mundial, debido a que se asocia con altas tasas de mortalidad, aumento en los niveles de resistencia a otros antimicrobianos, elevación en el potencial de diseminación e incremento en los costos de atención en salud. Objetivo: Caracterizar bacilos gramnegativos multirresistentes, aislados en pacientes hospitalizados en instituciones de salud de Barranquilla (Colombia). Material y Métodos: Estudio descriptivo acerca de la caracterización fenotípica y genotípica de la resistencia bacteriana en las infecciones asociadas a la atención en salud, mediada por carbapenemasas en aislados bacterianos enviados por los laboratorios pertenecientes a la red de laboratorios del Departamento del Atlántico. Resultados: La KPC fue la carbapenemasa más frecuente en las Enterobacterales $(27,6 \%)$, predominando en Klebsiella pneumoniae $(13,1 \%)$ sola y asociada a otras carbapenemasas. En Pseudomonas aeruginosa predominó la carbapenemasa VIM $(32,8 \%)$ y la OXA en Acinetobacter baumannii $(17,1 \%)$. Conclusión: Se encontró una amplia distribución de cepas multi-resistentes productoras de carbapenemasas en instituciones de salud de Barranquilla, las cuales expresaron los siguientes mecanismos de resistencia: KPC, VIM, NDM, OXA.

Palabras clave: Enterobacterales; Pseudomonas aeruginosa; Acinetobacter baumannii; carbapenemasas; carbapenems; $\beta$-lactámicos.

\section{Abstract}

Background: The emergence of carbapenem resistant gramnegative bacilli has become a problem of public health worldwide, because it is associated with high mortality rates, increased levels of resistance to other antimicrobials, increased potential for dissemination transition and increase in health care costs. Aim: To characterize multiresistant gram-negative bacilli, isolated in patients hospitalized in health institutions of Barranquilla (Colombia). Methods: A descriptive study was conducted on the phenotypic and genotypic characterization of bacterial resistance in infections associated with health care, mediated by carbapenemases in bacterial isolates sent by laboratories belonging to the laboratory network of the Department of Atlántico. Results: KPC was the most frequent carbapenemase in Enterobacterales (27.6\%), predominantly in Klebsiella pneumoniae (13.1\%) alone and associated with other carbapenemases. In Pseudomonas aeruginosa, VIM carbapenemase (32.8\%) predominated and OXA in Acinetobacter baumannii (17.1\%). Conclusion: A wide distribution of multi-resistant strains producing carbapenemases in Atlantic health institutions was found, which expressed the following resistance mechanisms: KPC, VIM, NDM, OXA.

Keywords: Enterobacterales; Pseudomonas aeruginosa; Acinetobacter baumannii; carbapenemases; carbapenems; betalactams.

\section{Correspondencia a:}

Ronald Maestre Serrano

rmaestre5@unisimonbolivar.edu.co 


\section{Introducción}

$\mathrm{E}$ 1 surgimiento de bacilos gramnegativos resistentes a carbapenémicos, es una grave amenaza para la salud de los pacientes y para la salud pública, porque se asocia a altas tasas de mortalidad, crecimiento en los grados de resistencia a otros antimicrobianos y un amplio potencial de diseminación ${ }^{1-3}$.

Los carbapenémicos son una clase de antimicrobianos $\beta$-lactámicos, que se han usado en los últimos años con mayor frecuencia, como parte de la terapéutica empírica inicial en diversas infecciones ${ }^{2} \mathrm{y}$ su uso repetido ha incrementado la resistencia, principalmente a Pseudomonas spp y Acinetobacter baumannii; los cuales, junto a varias Enterobacterales como Klebsiella spp, Escherichia coli, Serratia spp y Proteus spp fueron incluidos en la lista de patógenos de prioridad crítica en la resistentes a los antimicrobianos por la Organización Mundial de la Salud (OMS) ${ }^{4}$. Son bacterias que pueden provocar infecciones graves y a menudo letales, como bacteriemias y neumonías ${ }^{4}$.

En estos microorganismos pueden estar incluidos múltiples mecanismos de resistencia como la producción de carbapenemasas, las que hidrolizan a las penicilinas, cefalosporinas y en varios grados a los carbapenémi$\cos ^{5,6}$. Se han documentado en diversas partes del mundo brotes epidémicos producidos por Enterobacterales productoras de carbapenemasas, por lo general del tipo Klebsiella pneumoniae carbapenemasa (KPC) $)^{5,7}$. Los estudios moleculares de las carbapenemasas han identificado que éstas se encuentran en elementos móviles fácilmente transmisibles, lo que resalta la importancia epidemiológica de este nuevo mecanismo de resistencia ${ }^{6,8}$

Teniendo en cuenta lo anterior, la OMS prioriza la resistencia a los antimicrobianos en la generación de políticas y programas. Apoyando esta iniciativa, en Colombia se han realizado esfuerzos a través de la implementación de normas e inversión económica y de recurso humano. Además, se han creado redes, con el fin de recolectar de forma sistemática la información de resistencia bacteriana de cada institución y así plantear intervenciones acordes con cada uno; sin embargo, en el Departamento del Atlántico, no se dispone en la actualidad de datos consolidados que describan los principales mecanismos de resistencia de bacterias que afectan los pacientes en sus instituciones de salud.

\section{Objetivo}

Caracterizar bacilos gramnegativos multi-resistentes, aislados en pacientes hospitalizados en instituciones de salud del Departamento del Atlántico, que permitan diseñar estrategias para la toma de decisiones en su terapéutica.

\section{Métodos}

Se realizó un estudio descriptivo, retrospectivo y transversal en el que se analizaron 279 aislados de Enterobacterales y bacilos gramnegativos no fermentadores (BGNNF) tipo Pseudomonas aeruginosa y A. baumannii en instituciones de salud del distrito de Barranquilla y su área metropolitana durante los años 2012 a 2017 , que cumplían con los siguientes criterios de inclusión: resistencia disminuida al menos a un carbapenémico: imipenem (IPM), meropenem (MRP) o ertapenem (ETP), definida por la formación de halos de inhibición $\leq 21 \mathrm{~mm}$ y $C I M \geq 2 \mu \mathrm{g} / \mathrm{mL}$ para ertapenem, CIM $\geq 4 \mu \mathrm{g} / \mathrm{mL}$ para meropenem, imipenem y doripenem y resistencia a una cefalosporina de tercera y cuarta generación: ceftazidima (CAZ), ceftriaxona (CRO), cefotaxima (CTX) y cefepime (FEP) para Enterobacterales; y resistencia a ceftazidima y uno o más carbapenémicos para BGNNF, mediante antibiograma del equipo automatizado o por Kirby Bauer (KB) y pruebas de confirmación fenotípica.

La identificación y el estudio de susceptibilidad fueron efectuados por los laboratorios participantes utilizando equipos semi-automatizados. Posteriormente, fueron confirmados por el Laboratorio de Salud Pública (LSP) del Departamento del Atlántico por pruebas bioquímicas convencionales y la determinación de la susceptibilidad in vitro por el método de difusión de disco $\mathrm{KB}$ del Instituto para la Estandarización de los Laboratorios Clínicos (CLSI). Se realizó la detección de serin-carbapenemasas y metalo$\beta$-lactamasa (MBL) con las pruebas fenotípicas de test de Hodge modificado (TMH), ácido fenilborónico (en inglés APB) y ácido etilen-diamino-tetraacético/ácido mercaptoacético (en inglés EDTA/SMA) para Enterobacterales, y para los BGNNF se realizó EDTA/SMA. Para la prueba de APB y TMH, se utilizaron las cepas ATCC control de E. coli 25922, K. pneumoniae ATCC BAA 1705 (control positivo para KPC) у K. pneumoniae ATCC BAA 1706 (control negativo), y para la prueba de EDTA/SMA se utilizó la cepa control de $K$. pneumoniae ATCC BAA 2146 (control positivo para nueva delhi metalo- $\beta$-lactamasa-NDM).

La identificación de las carbapenemasas clínicamente más relevantes fue efectuada a 35,4\% (99/279) de las cepas estudiadas, a través de la técnica de reacción de polimerasa en cadena (RPC). De éstas, $(76,8 \%)$ que correspondían a 76 cepas resultaron confirmatorias para alguna de las diferentes $\beta$-lactamasas.

Para la detección de genes codificantes de carbapenemasas se utilizaron técnicas moleculares como estándar de oro; para los genes $b l a_{\mathrm{KPC}}, b a_{\mathrm{GES}}$ y $b l a_{\mathrm{VIM}}{ }^{9}$ se utilizó RPC múltiple y para $b l a_{\mathrm{NDM}}{ }^{10}$, bla ${ }_{\mathrm{IMP}}{ }^{11}$ y $b l a_{\text {OXA-48 }}{ }^{12}$ una RPC simple. La presencia de carbapenemasas del tipo OXA de los subgrupos bla $a_{\text {OXA-23 }}$, bla $a_{\text {OXA-24/40 }}, b l a_{\text {OXA-51 }}$, bla ${ }_{\text {OXA }-58}{ }^{13}$ y $b l a_{\text {OXA-143 }}{ }^{14}$ en $A$. baumannii, fue evaluada mediante RPC múltiple. 
del total de las muestras se aislaron en el distrito de Barranquilla y el 19,7\% restante en el municipio de Puerto Colombia ubicado en su área metropolitana.

Con respecto a las características demográficas de los pacientes en los que se realizaron los aislamientos bacterianos, 59,8\% (IC95\%: 53,8-65,6) de los casos eran hombres, la edad promedio fue de 50,4 años $\pm 25,7$; el paciente más joven era un lactante bajo un año de edad y el de mayor edad era de 98 años.

De los 279 aislados de bacterias multi-resistentes recibidos en el período estudiado, $142(50,8 \%)$ correspondieron a Enterobacterales, 135 (48,3\%) a BGNNF tipo $P$. aeruginosa y $A$. baumannii y dos $(0,7 \%)$ a cepas de Acinetobacter lwoffii y Acinetobacter spp (una de cada una). El microorganismo más frecuentemente aislado correspondió a $P$. aeruginosa (34,7\%), seguido de $K$. pneumoniae $(29,3 \%)$, A. baumannii $(13,6 \%)$, E. coli (9,6\%) y Enterobacter cloacae (6,4\%).

En los servicios No UCI se encontró el 44,1\% de los aislados y en las UCI el $38 \%$. En las salas No UCI la frecuencia de Enterobacterales fue de 58,6\% (n: 72) y BGNNF fue de 41,4\% (n: 51) siendo $P$. aeruginosa $(33,3 \%)$, K. pneumoniae $(30,9 \%)$ y E. coli $(10,5 \%)$ las más frecuente. En las salas UCI se observó una frecuencia de Enterobacterales de 40 (37,0\%) y de BGNNF de 68 (62,9\%); donde $P$. aeruginosa y $A$. baumannii presentaron frecuencia de 38,8 y $22,2 \%$ respectivamente (Tabla 1 ). durante el año 2016 con 77 cepas $(27,5 \%)$. El 80,2\%

\begin{tabular}{|c|c|c|c|c|c|}
\hline Bacterias gramnegativas aisladas & $\begin{array}{c}\text { No UCI }(n=123) \\
n(\%)\end{array}$ & $\begin{array}{c}\mathrm{UCI}(\mathrm{n}=108) \\
n(\%)\end{array}$ & $\begin{array}{c}\text { SD }(n=48) \\
n(\%)\end{array}$ & $\begin{array}{c}\text { Total }(n=279) \\
n(\%)\end{array}$ & IC (95\%) \\
\hline Acinetobacter Iwoffii & - & $1(0,9)$ & - & $1(0,3)$ & $0,0-1,9$ \\
\hline Acinetobacter baumannii & $10(8,1)$ & $24(22,2)$ & $4(8,3)$ & $38(13,6)$ & $9,8-18,2$ \\
\hline Acinetobacter spp. & - & $1(0,9)$ & - & $1(0,3)$ & $0,0-1,9$ \\
\hline Citrobacter freundii & $1(0,8)$ & - & - & $1(0,3)$ & $0,0-1,9$ \\
\hline Enterobacter aerogenes & $2(1,6)$ & - & - & $2(0,7)$ & $0,0-2,5$ \\
\hline Enterobacter cloacae & $12(9,7)$ & $1(0,9)$ & $5(10,4)$ & $18(6,4)$ & $3,8-10,0$ \\
\hline Enterobacter gergoviae & - & $1(0,9)$ & - & $1(0,3)$ & $0,0-1,9$ \\
\hline Escherichia coli & $13(10,5)$ & $7(6,4)$ & $7(14,5)$ & $27(9,6)$ & $6,4-13,7$ \\
\hline Klebsiella oxytoca & $2(1,6)$ & $1(0,93)$ & $0(0)$ & $3(1,0)$ & $0,2-3,1$ \\
\hline Klebsiella pneumoniae & $38(30,8)$ & $28(25,9)$ & $16(33,3)$ & $82(29,3)$ & $24,1-35,1$ \\
\hline Proteus mirabilis & $2(1,6)$ & - & - & $2(0,7)$ & $0,0-2,5$ \\
\hline Providencia rettgeri & $1(0,8)$ & - & $1(2,0)$ & $2(0,7)$ & $0,0-2,5$ \\
\hline Pseudomonas aeruginosa & $41(33,3)$ & $42(38,8)$ & $14(29,1)$ & $97(34,7)$ & $29,1-39,5$ \\
\hline Serratia fonticola & $1(0,8)$ & $0(0)$ & $0(0)$ & $1(0,3)$ & $0,0-1,9$ \\
\hline Serratia marcescens & $0(0)$ & $2(1,8)$ & $1(2,0)$ & $3(1,0)$ & $0,2-3,1$ \\
\hline
\end{tabular}


Las Enterobacterales y BGNNF más frecuente fueron aislados de muestras de orina (29,0\%, IC95\%: 23,7-34,7) en las cuales predominaron $K$. pneumoniae $(35,8 \%), P$. aeruginosa $(28,4 \%)$, E. coli $(17,3 \%)$ y E. cloacae $(12,3 \%)$; seguidas de muestras de secreciones obtenidas de vías respiratorias $(20,7 \%$, IC95\%: 16,1 -26,0) donde predominaron $P$. aeruginosa $(43,1 \%), K$. pneumoniae $(23,1 \%)$ y $A$. baumannii $(25,9 \%)$ y muestras de sangre $(20,0 \%$, IC $95 \%$ 15,5 - 25,2) con predominio de $K$. pneumoniae $(37,5 \%)$, $P$. aeruginosa (23,2\%) y A. baumannii (14,3\%) (Tabla 2).

El 76,8\% (76/99) de los aislados procesados por RPC se confirmaron como productoras de carbapenemasas y el $23,2 \%$ (23/99) restante, confirmadas como negativas por RPC, se clasificaron como resistentes a carbapenémicos por otros mecanismos que no involucran la producción de carbapenemasas.

En el grupo de las Enterobacterales se obtuvo 35 (46\%) de las carbapenemasas, de las cuales 21 (27\%) son KPC, cuatro NDM, tres VIM y las siguientes co-producciones: una KPC+VIM, cinco KPC+NDM y una NDM+GES. En K. pneumoniae predominó KPC $10(13,1 \%)$ sola y asociada a otras carbapenemasas $6(7,8 \%)$, se le identificaron tres VIM, tres NDM, una co-producción de $\mathrm{KPC}+\mathrm{VIM}$, cinco co-producciones de KPC+NDM y una combinación de NDM+GES. En E. coli y E. cloacae se obtuvieron cuatro KPC y seis KPC, respectivamente, y en dos cepas de Proteus mirabilis la enzima NDM y KPC, independientemente. En el resto de las Enterobacterales examinadas no se aislaron carbapenemasas.

En los BGNNF se encontró 41 (54,9\%) de las carbapenemasas: en $P$. aeruginosa la enzima VIM (32,8\%), dos KPC y una co-producción de KPC+VIM. En los aislados de $A$. baumannii, se identificaron dos cepas con la combinación de NDM+OXA-51, una de VIM+OXA-23 y 10 productoras de OXA-23+OXA-51, predominando esta última. No se identificó KPC en este patógeno (Tabla 3).

La mayor frecuencia de las carbapenemasas se registró en hombres $(67,1 \%)$. No se encontró asociación entre el sexo de los pacientes y patógenos bacterianos productores de carbapenemasas $[\mathrm{RR}=0,73$; IC 95\%: $0,48-1,10 ;(p>0,05)]$. Las fuentes de aislamiento más frecuente de bacterias productoras de carbapenemasas fueron la orina, sangre, el sistema respiratorio y los tejidos blandos No se encontró relación de dependencia entre las carbapenemasas y su fuente de aislamiento $(p>0,05)$ (Tabla 4).

La frecuencia de carbapenemasas en muestras procedentes de UCI fue de $29 \%$ (22/76) y de otros servicios no UCI fue de 44,7\% (34/76). No se identificó el servicio en $26,3 \%$ de las muestras; así mismo, no se encontró relación de dependencia entre la presencia de carbapenemasa y los servicios hospitalarios $(\mathrm{p}>0,05)$ (Tabla 5).

\begin{tabular}{|c|c|c|c|c|c|c|c|c|}
\hline Tipo de muestra & $\begin{array}{l}\text { P. aeruginosa } \\
(\mathrm{n}=94) \\
\mathrm{n}(\%)\end{array}$ & $\begin{array}{c}\text { K. pneumoniae } \\
(\mathrm{n}=85) \\
\mathrm{n}(\%)\end{array}$ & $\begin{array}{l}\text { A. baumannii } \\
(\mathrm{n}=38) \\
n(\%)\end{array}$ & $\begin{array}{c}\text { E. coli } \\
(n=27) \\
n(\%)\end{array}$ & $\begin{array}{c}\text { E. cloacae } \\
(n=18) \\
n(\%)\end{array}$ & $\begin{array}{l}\text { Otras bacterias } \\
\qquad(\mathrm{n}=17) \\
\mathrm{n}(\%)\end{array}$ & $\begin{array}{c}\text { Total } \\
(n=279) \\
n(\%)\end{array}$ & IC (95\%) \\
\hline Sistema renal & $23(28,4)$ & $29(35,6)$ & 0 & $14(17,2)$ & $10(12,3)$ & $5(6,1)$ & $81(29,0)$ & $23,7-34,7$ \\
\hline Vías respiratorias & $25(43,1)$ & $14(23,1)$ & $15(25,9)$ & $1(1,23)$ & $1(1,2)$ & $2(2,5)$ & $58(20,8)$ & $16,1-26,0$ \\
\hline Sangre & $13(23,2)$ & $21(37,5)$ & $8(14,3)$ & $3(5,35)$ & $3(5,4)$ & $8(14,2)$ & $56(20,0)$ & $15,5-25,2$ \\
\hline Tejidos blandos & $9(37,5)$ & $3(12,5)$ & $5(20,8)$ & $4(16,6)$ & $1(4,1)$ & $2(8,2)$ & $24(8,6)$ & $5,5-12,5$ \\
\hline Secreción abdominal & $4(21,0)$ & $7(36,8)$ & $3(15,7)$ & $3(15,8)$ & $2(10,5)$ & - & $19(6,8)$ & $4,1-10,4$ \\
\hline Secreción de escara & $2(28,0)$ & $1(14,0)$ & $2(28)$ & $1(14)$ & $1(14)$ & - & $7(2,5)$ & $1,0-5,1$ \\
\hline Catéter & $2(40,0)$ & $1(20,0)$ & $2(40)$ & - & - & - & $5(1,8)$ & $0,5-4,1$ \\
\hline Secreción de oído & $4(80,0)$ & $1(20,0)$ & - & - & - & - & $5(1,8)$ & $0,5-4,1$ \\
\hline Secreción de herida & $1(20,0)$ & $3(60,0)$ & $1(20)$ & - & - & - & $5(1,8)$ & $0,5-4,1$ \\
\hline Sin datos & $2(40,0)$ & $1(20,0)$ & $1(20)$ & $1(20)$ & - & - & $5(1,8)$ & $0,5-4,1$ \\
\hline Líquidos corporales & $4(100)$ & - & - & - & - & - & $4(1,4)$ & $0,3-3,6$ \\
\hline Secreción de pierna & $1(33,3)$ & $2(66,6)$ & - & - & - & - & $3(1,1)$ & $0,2-3,1$ \\
\hline Secreción ocular & $2(100)$ & - & - & - & - & - & $2(0,7)$ & $0,0-2,5$ \\
\hline Úlcera & $1(50,0)$ & $1(50,0)$ & - & - & - & - & $2(0,7)$ & $0,0-2,5$ \\
\hline Secreción de cadera & - & $1(100)$ & - & - & - & -0 & $1(0,4)$ & $0,0-1,9$ \\
\hline Secreción de tumor en la cara & $1(100)$ & - & - & - & - & - & $1(0,4)$ & $0,0-1,9$ \\
\hline Secreción de piel & - & - & $1(100)$ & - & - & - & $1(0,4)$ & $0,01-1,98$ \\
\hline
\end{tabular}


Tabla 3. Identificación de carbapenemasas en bacilos gramnegativos multi-resistentes, aislados en instituciones de salud del distrito de Barranquilla y su área metropolitana durante los años 2012-2017

\begin{tabular}{|c|c|c|c|c|c|c|c|c|}
\hline Tipo de enzima & $\begin{array}{c}\text { K. pneumoniae } \\
\text { n (\%) }\end{array}$ & $\begin{array}{l}\text { P. mirabilis } \\
\text { n (\%) }\end{array}$ & $\begin{array}{c}\text { E. cloacae } \\
\text { n }(\%)\end{array}$ & $\begin{array}{l}\text { E. coli } \\
\text { n }(\%)\end{array}$ & $\begin{array}{c}\text { Total Enterobacterales } \\
\text { n (\%) }\end{array}$ & $\begin{array}{c}\text { P. aeruginosa } \\
\text { n (\%) }\end{array}$ & $\begin{array}{c}\text { A. baumannii } \\
\text { n (\%) }\end{array}$ & $\begin{array}{l}\text { Total } \\
\text { n } \quad(\%)\end{array}$ \\
\hline $\mathrm{KPC}$ & $10(13,1)$ & $1(1,3)$ & $6(7,8)$ & $4(5,2)$ & $21(27,6)$ & $2(2,6)$ & - & $23 \quad(30,2)$ \\
\hline KPC-NDM & $5(6,5)$ & - & - & - & $5(6,5)$ & - & - & $5 \quad(6,5)$ \\
\hline KPC-VIM & $1 \quad(1,3)$ & - & - & - & $1 \quad(1,3)$ & $1 \quad(1,3)$ & - & $(2,6)$ \\
\hline NDM & $3(4,0)$ & $1(1,3)$ & - & - & $4 \quad(5,2)$ & - & - & $(5,2)$ \\
\hline NDM-OXA 51 & - & - & - & - & - & - & $2(2,6)$ & $(2,6)$ \\
\hline NDM - GES & $1(1,3)$ & - & - & - & $1 \quad(1,3)$ & - & - & $(1,3)$ \\
\hline OXA $23+$ VIM & - & - & - & - & - & - & $1(1,3)$ & $1 \quad(1,3)$ \\
\hline OXA 23 - 51 & - & - & - & - & - & - & $10(13,1)$ & $10 \quad(13,1)$ \\
\hline VIM & $3(4,0)$ & - & - & - & $3(4,0)$ & $25(32,8)$ & - & $28 \quad(36,8)$ \\
\hline Total & $23(30,2)$ & $2(2,6)$ & $6(7,8)$ & $4(5,2)$ & $35(46,0)$ & $28(36,8)$ & $13(17,1)$ & $76(100)$ \\
\hline
\end{tabular}

n: frecuencia absoluta; \%: frecuencia relativa.

Tabla 4. Identificación de carbapenemasas en bacilos gramnegativos multi-resistentes según tipo de muestra

\begin{tabular}{|c|c|c|c|c|c|c|c|c|c|c|}
\hline Tipo de muestra & $\begin{array}{l}\text { KPC } \\
\text { n (\%) }\end{array}$ & $\begin{array}{c}\text { KPC-NDM } \\
\text { n (\%) }\end{array}$ & $\begin{array}{l}\text { KPC-VIM } \\
\text { n (\%) }\end{array}$ & $\begin{array}{l}\text { NDM } \\
\text { n (\%) }\end{array}$ & $\begin{array}{c}\text { NDM-OXA } 51 \\
\text { n (\%) }\end{array}$ & $\begin{array}{l}\text { NDM-GES } \\
\text { n (\%) }\end{array}$ & $\begin{array}{c}\text { Bla OXA 23-VIM } \\
\text { n (\%) }\end{array}$ & $\begin{array}{c}\text { Bla OXA 23-51 } \\
\text { n (\%) }\end{array}$ & $\begin{array}{l}\text { VIM } \\
\text { n (\%) }\end{array}$ & $\begin{array}{l}\text { Total } \\
\text { n (\%) }\end{array}$ \\
\hline Catéter & - & - & - & - & - & - & - & $1(1,31)$ & - & $1(1,31)$ \\
\hline Sangre & $8(10,52)$ & $2(2,63)$ & - & - & - & - & $1(1,31)$ & $2(2,63)$ & $2(2,63)$ & $15(19,73)$ \\
\hline SD & - & - & - & - & - & - & - & - & $1(1,31)$ & $1(1,31)$ \\
\hline Secreción abdominal & $3(3,94)$ & - & - & - & - & - & - & $1(1,31)$ & $3(3,94)$ & $7(9,21)$ \\
\hline Secreción de cadera & - & $1(1,31)$ & - & - & - & - & - & - & - & $1(1,31)$ \\
\hline Secreción de escara & - & - & - & - & - & - & - & - & $2(2,63)$ & $2(2,63)$ \\
\hline Secreción de herida & $1(1,3)$ & - & - & - & - & - & - & $1(1,31)$ & - & $2(2,63)$ \\
\hline Secreción ocular & - & - & - & - & - & - & - & - & $2(2,63)$ & $2(2,63)$ \\
\hline Secreción de pierna & - & - & - & - & - & - & - & - & $1(1,63)$ & $1(1,31)$ \\
\hline Sistema renal & $7(9,2)$ & $1(1,3)$ & $2(2,6)$ & $1(1,3)$ & 0 & $1(1,3)$ & 0 & 0 & $10(13,1)$ & $22(28,9)$ \\
\hline Tejidos blandos & $1(1,3)$ & 0 & 0 & $2(2,6)$ & 0 & 0 & 0 & $2(2,6)$ & $3(3,9)$ & $8(10,5)$ \\
\hline Vías respiratorias & $3(3,9)$ & $1(1,3)$ & 0 & $1(1,3)$ & $2(2,6)$ & 0 & 0 & $3(3,9)$ & $4(5,2)$ & $14(18,4)$ \\
\hline Total & $23(30,2)$ & $5(6,5)$ & $2(2,6)$ & $4(5,2)$ & $2(2,6)$ & $1(1,3)$ & $1(1,3)$ & $10(13,1)$ & $28(36,8)$ & $76(100)$ \\
\hline
\end{tabular}

Tabla 5. Identificación de carbapenemasas en bacilos gramnegativos multi-resistentes según servicio UCI y No UCI

\begin{tabular}{|c|c|c|c|c|c|c|c|c|c|c|}
\hline Servicio & $\begin{array}{c}\text { KPC } \\
\text { n (\%) }\end{array}$ & $\begin{array}{c}\text { KPC-NDM } \\
\text { n (\%) }\end{array}$ & $\begin{array}{l}\text { KPC-VIM } \\
\text { n (\%) }\end{array}$ & $\begin{array}{l}\text { NDM } \\
\text { n (\%) }\end{array}$ & $\begin{array}{c}\text { NDM-OXA } 51 \\
n(\%)\end{array}$ & $\begin{array}{c}\text { NDM-GES } \\
\text { n (\%) }\end{array}$ & $\begin{array}{c}\text { Bla OXA 23-VIM } \\
\text { n (\%) }\end{array}$ & $\begin{array}{c}\text { Bla OXA 23-51 } \\
\text { n (\%) }\end{array}$ & $\begin{array}{l}\text { VIM } \\
\text { n (\%) }\end{array}$ & $\begin{array}{l}\text { Total } \\
\text { n (\%) }\end{array}$ \\
\hline Consulta externa & $1(1,3)$ & - & - & - & - & - & - & $1(1,3)$ & $2(2,6)$ & $4(5,2)$ \\
\hline Cirugía & - & - & - & - & - & - & - & $1(1,3)$ & - & $1(1,3)$ \\
\hline Hospitalización & $5(6,5)$ & $4(5,2)$ & $1(1,3)$ & $2(2,6)$ & - & $1(1,32)$ & - & - & $10(13,1)$ & $23(30,2)$ \\
\hline Medicina Interna & $1(1,3)$ & - & - & - & - & - & - & - & - & $1(1,3)$ \\
\hline SD & $10(13,1)$ & - & - & - & - & - & $1(1,32)$ & $3(3,9)$ & $6(7,8)$ & $20(26,3)$ \\
\hline Sepsis & - & - & - & - & - & - & - & - & $1(1,3)$ & $1(1,3)$ \\
\hline $\mathrm{UCl}$ & $6(7,8)$ & - & $1(1,3)$ & $1(1,3)$ & $2(2,6)$ & - & - & $5(6,5)$ & $7(9,2)$ & $22(28,9)$ \\
\hline Urgencias & - & $1(1,3)$ & - & $1(1,3)$ & - & - & - & - & $2(2,6)$ & $4(5,2)$ \\
\hline Total & $23(30,2)$ & $5(6,5)$ & $2(2,6)$ & $4(5,2)$ & $2(2,6)$ & $1(1,3)$ & $1(1,3)$ & $10(13,1)$ & $28(36,8)$ & $76(100)$ \\
\hline
\end{tabular}




\section{Discusión}

En el presente estudio se encontró a $P$. aeruginosa, $K$. pneumoniae y $A$. baumannii como los patógenos aislados con máyor frecuencia, independientemente del servicio clínico de origen, microorganismos oportunistas reconoci$\operatorname{dos}^{15-17}$, que afectan principalmente a los pacientes hospitalizados y permanecen además en superficies inanimadas por largos período, facilitando su diseminación ${ }^{18}$. Este hallazgo es concordante con estudios previos en hospitales y clínicas de Latinoamérica incluida Colombia ${ }^{16,17,19}$. Algunas diferencias con otros estudios colombianos que citamos a continuación podrían atribuirse a un sub-registro de datos de procedencia de la muestra según servicios. El primero de dichos estudios que difiere de nuestros resultados fue realizado entre los años 2009-2012 en UCIs de instituciones de salud de Colombia, siendo $E$. coli el microorganismo más frecuente seguido de $P$. aeruginosa y A. baumannii. Un segundo estudio, tipo vigilancia, realizado entre los años 2012-2014, reportó a $K$. pneumoniae y E. coli como las Enterobacterales más frecuentes en UCI y a $E$. coli seguida de $K$. pneumoniae y $P$. aeruginosa en otras áreas de hospitalización. El tercer estudio realizado en una UCI de una institución de salud de la ciudad de Montería indicó la prevalencia de A. baumannii, $K$. pneumoniae, $P$. aeruginosa y E. coli y un cuarto estudio realizado en una UCI de una institución de salud del municipio de Pereira encontró a $E$. coli, $K$. pneumoniae y $P$. aeruginosa como los microorganismos más frecuentemente aislados ${ }^{20-22}$.

En otros países de Latinoamérica se ha reportado a $P$. aeruginosa y $A$. baumannii como los microorganismos aislados con mayor frecuencia en pacientes de UCI y Unidad de Cuidados Intermedios-UCIN ${ }^{23,24}$; todos estos estudios demuestran la diferencias que existe en la epidemiología entre instituciones, ciudades y países, debido a las condiciones de microbiología según el tipo de pacientes que reciben, el patrón de uso de antimicrobianos y condiciones ambientales de la región ${ }^{16}$.

En el período observado en nuestro estudio, se identificó una amplia distribución de las carbapenemasas en las instituciones de salud participantes de la ciudad de Barranquilla, de tipo KPC, seguida de NDM en Enterobacterales; VIM y KPC en $P$. aeruginosa y OXA en $A$. baumannii; estos resultados concuerdan con otros estudios reportados en Colombia ${ }^{21,25}$. A nivel mundial se observa un comportamiento similar, encontrándose que en $P$. aeruginosa las carbapenemasas más detectadas son las del tipo de las metalo- $\beta$-lactamasas ${ }^{5,26}$. Estos hallazgos coinciden con los de otras investigaciones extranjeras, donde $K$. pneumoniae es una de las Enterobacterales que más frecuentemente se aísla de infecciones nosocomiales y es la especie que se asocia con mayor prevalencia a las carbapenemasas tipo $\mathrm{KPC}^{27}$; sin embargo, en otros países de Sudamérica se han reportado hallazgos diferentes con predominancia de carbapenemasas tipo NDM y en países de Europa como España la predominancia de carbapenemasas tipo OXA-48 ${ }^{19,28}$.

De KPC se reconocen actualmente 23 variantes de las cuales KPC-1, fue la primera variante descrita en el 2001, en un aislado de K. pneumoniae en Carolina del Norte ${ }^{29}$. En Sudamérica, el primer reporte de enzimas KPC fue efectuado en Colombia en el año 2005, detectando la variante $\mathrm{KPC}-2$ en dos aislados de $K$. pneumoniae de diferentes hospitales de Medellín ${ }^{9,30}$. Posteriormente, en el 2007, en esta misma ciudad apareció el primer reporte en el mundo de KPC-2 en P. aeruginos $a^{30}$. En el presente informe, KPC fue la carbapenemasa más frecuentemente detectada en $K$. pneumoniae, lo cual coincide con lo reportado en otras comunicaciones ${ }^{31-33}$. Actualmente, se considera que dada la diseminación de las carbapenemasas KPC-2 y KPC-3, Colombia es endémica para estas enzimas con una frecuencia que alcanza el 70,3\% en especies de Enterobacterales ${ }^{16,21}$.

Con relación a la presencia de metalo- $\beta$-lactamasas tipo VIM, en nuestro estudio, ésta se aisló en mayor frecuencia en $P$. aeruginosa y K. pneumoniae. Se han descrito más de 46 variantes de la enzima VIM-1, detectada por primera vez en Italia, en un aislado de $P$. aeruginosa ${ }^{34}$. En Latinoamérica, se ha reportado el aislamiento de esta misma especie en Colombia, Chile, Venezuela, Brasil y Argentina ${ }^{35}$.

Otra metalo- $\beta$-lactamasa hallada en nuestro estudio fue la NDM en dos cepas de $A$. baumannii en co-producción con OXA-51, aislada en vías respiratorias, e identificada también en $K$. pneumoniae y $P$. mirabilis. La enzima NDM se reportó por primera vez en el año 2008 en Nueva Delhi, en un aislado de K. pneumoniae y $E$. coli $i^{36}$ y posteriormente se reportó en diferentes países de Asia, Europa, África y Norteamérica ${ }^{37}$. En Colombia, la enzima NDM-1 se detectó por primera vez en $K$. pneumoniae en un brote que afectó pacientes de una unidad neonatal en Bogotá y posteriormente se identificó en otras ciudades del país ${ }^{38}$.

En nuestros resultados se detectó GES (del inglés Guyana extended spectrum) en una cepa de K. pneumoniae. Actualmente, se reconocen más de 27 variantes de enzimas GES que se han reportado en aislados de $P$. aeruginosa, $K$. pneumoniae, E. coli y Serratia marcescens en países de Latinoamérica ${ }^{39}$. En Colombia, se reportó por primera vez en aislados de Citrobacter spp., Enterobacter spp., y Pseudomonas spp., así como la producción simultánea de KPC más GES y de VIM más GES ${ }^{21}$.

Además, encontramos más frecuentemente la OXA-23 en co-producción con OXA-51 en $A$. baumannii; estos mismos resultados se han encontrado en otros estudios colombianos; adicionalmente se ha identificado las carbapenemasas OXA-143, OXA-58 y la OXA-72 del subgrupo OXA-24/40 38 .

Mundialmente se han incrementado los casos de co- 
están basadas en la capacidad de ciertas sustancias de inhibir estas enzimas permitiendo hacer un diagnóstico presuntivo de la presencia de carbapenemasas en aislados clínicos, lo cual es importante desde el punto de vista terapéutico, epidemiológico y de control. Por tanto, las cepas no procesadas por RPC y con pruebas fenotípicas negativas se clasificaron resistentes por otros mecanismos que no involucraban carbapenemasas y las positivas como posibles productores de carbapenemasas, tipo serin o metalo- $\beta$-lactamasa. Durante el estudio las pruebas rápidas carbaNP o sus variantes carbaACINETO o carbaBLUE no estaban incluidas en el protocolo del INS para la vigilancia de la resistencia bacteriana; éstas fueron implementadas posteriormente.

En conclusión, se encontró una amplia distribución de las carbapenemasas en las muestras analizadas, siendo la más frecuente VIM $(36,8 \%)$ en $P$. aeruginosa, seguida por KPC $(30,2 \%)$ en $K$. pneumoniae y las combinaciones OXA-23-OXA-51 (13,5\%) en. A baumannii, aisladas con mayor frecuencia del sistema renal, respiratorio y sangre.

Agradecimientos. A las instituciones de salud que pertenecen a la red departamental de microbiología del Departamento del Atlántico, al Instituto Nacional de Salud de Colombia por la confirmación de las cepas, y finalmente, al recurso humano del laboratorio de microbiología del Laboratorio de Salud Pública del Departamento del Atlántico.

\section{Referencias bibliográficas}

1.- Meletis G. Carbapenem resistance: overview of the problem and future perspectives. Ther Adv Infect Dis. 2016; 3 (1): 15-21. doi: 10.1177/2049936115621709.

2.- Kelly A M, Mathema B, Larson E L. Carbapenem-resistant Enterobacteriaceae in the community: a scoping review. Int $\mathrm{J}$ Antimicrob Agents [Internet]. 2017; 50 (2): 127-34. Available from: http://dx.doi. org/10.1016/j.ijantimicag.2017.03.012.

3.- Centers for Diasease Control and Prevention. CDC. Facility guidance for control of carbapenem-resistant Enterobacteriaceae (CRE) [Internet]. National Center for Emerging and Zoonotic Infectious Diseases. 2015. p. 24. Available from: https://www.osha.gov/SLTC/ ebola/control_prevention.html.

4.- Venter H. Reversing resistance to counter antimicrobial resistance in the World Health Organisation's critical priority of most dangerous pathogens. Biosci Rep. 2019; 29 (4): 1-12. doi: 10.1042/BSR20180474.

5.- Queenan A M, Bush K. Carbapenemases: The versatile $\beta$-lactamases. Clin Microbiol Rev. 2007; 20 (3): 440-58. doi: 10.1128/ CMR.00001-07.
6.- Gupta N, Limbago B M, Patel J B, Kallen A J. Carbapenem-resistant Enterobacteriaceae: epidemiology and prevention. Clin Infect Dis. 2011; 53 (1): 60-7. doi: $10.1093 / \mathrm{cid} / \mathrm{cir} 202$.

7.- Jia C C, Hong W Z, Zhang R, Chen G X. Emergence of Serratia marcescens, Klebsiella pneumoniae, and Escherichia coli isolates possessing the plasmid-mediated carbapenemhydrolyzing $\beta$-lactamase KPC- 2 in intensive care units of a chinese hospital. Antimicrob Agents Chemother. 2008; 52 (6): 2014-8. doi: 10.1128/AAC.01539-07.

8.- Magiorakos A, Srinivasan A, Carey R B, Carmeli Y, Falagas M E, Giske C G, et al. Multidrug-resistant, extensively drugresistant and pandrug-resistant bacteria: an International Expert Proposal for Interim Standard Definitions for Acquired Resistance. Clin Microbiol Infect 2012; 2012; 18: 268-81. doi: 10.1111/j.1469-0691.2011.03570.x.

9.- Monteiro J, Widen R H, Pignatari A C C, Kubasek C, Silbert S. Rapid detection of carbapenemase genes by multiplex real-time PCR. J Antimicrob Chemother. 2012; 67 (4): 906-9. doi: $10.1093 / \mathrm{jac} / \mathrm{dkr} 563$.

10.- Bonnin R A, Naas T, Poirel L, Nordmann P. Phenotypic, biochemical, and molecular techniques for detection of metallo- $\beta$-lactamase NDM in Acinetobacter baumannii. J Clin Microbiol. 2012; 50 (4): 1419-21. doi: 10.1128/ JCM.06276-11.

11.- Garza-Ramos U, Morfin-Otero R, Sader H S, Jones R N, Hernández E, Rodriguez-Noriega $\mathrm{E}$, et al. Metallo- $\beta$ lactamase gene blaIMP-15 in a class 1 integron, In95, from Pseudomonas aeruginosa clinical isolates from a hospital in Mexico. Antimicrob Agents Chemother. 2008; 52 (8): 2943-6. doi: 10.1128/AAC.00679-07.

12.- Poirel L, Walsh T R, Cuvillier V, Nordmann P. Multiplex PCR for detection of acquired carbapenemase genes. Diagn Microbiol Infect Dis [Internet]. 2011; 70 (1): 119-23. Available from: http://dx.doi.org/10.1016/j. diagmicrobio.2010.12.002.

13.- Woodford N, Ellington M J, Coelho J M, Turton J F, Ward M E, Brown S, et al. Multiplex PCR for genes encoding prevalent OXA carbapenemases in Acinetobacter spp. Int J Antimicrob Agents. 2006; 27 (4): 351-3. doi: 10.1016/j.ijantimicag.2006.01.004.

14.- Higgins P G, Lehmann M, Seifert $H$. Inclusion of OXA-143 primers in a multiplex polymerase chain reaction (PCR) for genes encoding prevalent OXA carbapenemases 


\section{—}

in Acinetobacter spp. Int J Antimicrob Agents. 2010; 35 (3): 305. doi: 10.1016/j. ijantimicag.2009.10.014.

15.- Marchaim D, Chopra T, Pogue J M, Pérez F, Hujer A M, Rudin S, et al. Outbreak of colistin-resistant, carbapenem-resistant Klebsiella pneumoniae in Metropolitan Detroit, Michigan. Antimicrob Agents Chemother. 2011; 55 (2): 593-9. doi: 10.1128/AAC.01020-10.

16.- Gales A C, Castanheira M, Jones R N, Sader H S. Antimicrobial resistance among Gramnegative bacilli isolated from Latin America: Results from SENTRY Antimicrobial Surveillance Program (Latin America, 20082010). Diagn Microbiol Infect Dis [Internet]. 2012; 73 (4): 354-60. Available from: http:// dx.doi.org/10.1016/j.diagmicrobio.2012.04.007.

17.- Rodríguez A P V, Ortega M H D, Garzón L I B, Vargas S M R, Iguarán D E H, Botero M V V, et al. Trends of bacterial resistance phenotypes in high-complexity public and private hospitals in Colombia. Rev Panam Salud Pública. 2011; 30 (6): 627-33. https://www.paho.org/hq/ dmdocuments/2014/Pan-American-JournalRes-Antim-2011.pdf.

18.- Rivera-Jacinto M A. Betalactamasas de espectro extendido en cepas de Escherichia coli y Klebsiella sp. aisladas de reservorios inanimados en un hospital del norte del Perú. Rev Esp Quimioter. 2012; 25 (2): 161-3. https://seq.es/wp-content/uploads/2012/06/seq. es_seq_0214-3429_25_2_rivera.pdf.

19.- Eddie Angles-Yanqui, Jorge Huaringa-Marcelo, Sacsaquispe-Contreras R, Luis Y, PampaEspinoza. Panorama de las carbapenemasas en Perú. Rev Panam Salud Publica [Internet]. 2020; 44: 1-10. Available from: https://doi. org/10.26633/RPSP.2020.61.

20.- Hernández-Gómez C, Blanco V M, Motoa G, Correa A, Maya J J, de la Cadena E, et al. Evolución de la resistencia antimicrobiana de bacilos Gram negativos en unidades de cuidados intensivos en Colombia. Biomedica. 2014; 34 (Suppl.1): S91-S100. doi: https://doi. org/10.7705/biomedica.v34i0.1667.

21.- Ovalle M V, Saavedra S Y, González M N, Hidalgo A M, Duarte C, Beltran M. Resultados de la vigilancia nacional de la resistencia antimicrobiana de enterobacterias y bacilos Gram negativos no fermentadores en infecciones asociadas a la atención de salud, Colombia, 2012-2014. Biomedica. 2017; 37: 473-85. doi: https://doi.org/10.7705/biomedica. v37i4.3432.

22.- Guzmán-terán C, Grupo M S, Farmacéuticas D I, Facultad I, De D C. Análisis de usos y resistencia a antibióticos en una UCI de Montería, Colombia. Rev Méd Risaralda. 2018; 24 (2): 75-80. doi: https://doi. org/10.22517/25395203.18571.

23.- Cusicanqui Luis Ángel C, Vega Juan R, Arrasco Rober Andre P, Campos Neftali RESUMEN
R, Ruiz Gallo Lambayeque P, Residente M. Infección nntrahospitalaria por bacterias gram negativas no fermentadoras en los pacientes hospitalizados en los servicios de UCI-UCIN del Hospital Regional Lambayeque 2014. Rev Exp Med. 2015; 1 (2): 56-60. https://siga. regionlambayeque.gob.pe/hrl_investigacion/ pdf/jul-dic-2015.pdf.

24.- Aguirre-Ávalos G, Mijangos-Méndez J C, Zavala-Silva M L, Coronado-Magaña $\mathrm{H}$, Amaya-Tapia G. Bacteremia por Acinetobacter baumannii en pacientes en estado crftico. Gac Med Mex. 2009; 145 (1): 21-5. https:// www.medigraphic.com/pdfs/gaceta/gm-2009/ gm091d.pdf.

25.- Saavedra S Y, Duarte C, González M N, Realpe M E. Caracterización de aislamientos de Pseudomonas aeruginosa productores de carbapenemasas de siete departamentos de Colombia. Biomedica. 2014; 34 (Suppl.1): S217-S223. doi: https://doi.org/10.7705/ biomedica.v34i0.1685.

26.- Nicolau C J, Oliver A. Carbapenemasas en especies del género Pseudomonas. Enferm Infecc Microbiol Clin [Internet]. 2010; 28 (Suppl. 1): S19-S28. Available from: http:// dx.doi.org/10.1016/S0213-005X(10)70004-5.

27.- González Rocha G, Vera Leiva A, Barría Loaiza C, Carrasco Anabalón S, Lima C, Aguayo Reyes A, et al. KPC: Klebsiella pneumoniae carbapenemasa, principal carbapenemasa en enterobacterias. Rev Chilena Infectol 2017; 34 (5): 476-84. http://dx.doi.org/10.4067/S071610182017000500476.

28.- Antequera M A, Sáez B C, Ciudad S M, García B M J, Moyano V B, Rodríguez C P, et al. Epidemiología, tratamiento y mortalidad en pacientes infectados por enterobacterias productoras de carbapenemasas: estudio retrospectivo. Rev Chilena Infectol 2020; 37 (3): 295-303. http://dx.doi.org/10.4067/s071610182020000300295

29.- Yigit H, Queenan A M, Anderson G J, Domenech-Sanchez A, Biddle J W, Steward C D, et al. Novel carbapenem-hydrolyzing $\beta$-lactamase, KPC-1, from a carbapenemresistant strain of Klebsiella pneumoniae. Antimicrob Agents Chemother. 2001; 45 (4): 1151-61. doi: 10.1128/AAC.45.4.11511161.2001

30.- Villegas M V, Lolans K, Correa A, Kattan J N, Lopez J A, Quinn J P. First identification of Pseudomonas aeruginosa isolates producing a KPC-type carbapenem-hydrolyzing $\beta$-lactamase. Antimicrob Agents Chemother. 2007; 51 (4): 1553-5. doi: 10.1128/AAC.0140506.

31.- Pacheco R, Osorio L, Correa A M, Villegas M V. Prevalence of Gram-negative bacteria harboring blaKPC gene in Colombian hospitals. Biomedica. 2014; 34 Suppl 1: S81-S90. doi: 10.1590/S0120-41572014000500010.
32.- Instituto de Salud Publica Programa de Control de Infecciones Asociadas a la Atencion en Salud. Boletin de Resistencia Antimicrobiana [Internet]. Boletín Instituto de Salud Pública de Chile. 2015. Available from: http://www.ispch. cl/sites/default/files/Boletin Gono-Final.pdf

33.- Lefebvre $B$, Lévesque $S$, Bourgault A M, Mulvey M R, Mataseje L, Boyd $\mathrm{D}$, et al. Carbapenem non-susceptible Enterobacteriaceae in Quebec, Canada: Results of a laboratory surveillance program (2010-2012). PLoS One. 2015; 10 (4): 1-14. doi: 10.1371/journal.pone. 0125076 .

34.- Lauretti L, Riccio M L, Mazzariol A, Cornaglia G, Amicosante G, Fontana R, et al. Cloning and characterization of bla(VIM), a new integron-borne metallo- $\beta$-lactamase gene from a Pseudomonas aeruginosa clinical isolate. Antimicrob Agents Chemother. 1999; 43 (7): 1584-90. doi: 10.1128/AAC.43.7.1584.

35.- Escandón-Vargas K, Reyes S, Gutiérrez S, Villegas M V. The epidemiology of carbapenemases in Latin America and the Caribbean. Expert Rev Anti Infect Ther [Internet]. 2017; 15 (3): 277-97. Available from: http://dx.doi.org/10.1080/14787210.2017 .1268918 .

36.- Yong D, Toleman M A, Giske C G, Cho H S, Sundman K, Lee K, et al. Characterization of a new metallo- $\beta$-lactamase gene, bla NDM- 1 , and a novel erythromycin esterase gene carried on a unique genetic structure in Klebsiella pneumoniae sequence type 14 from India. Antimicrob Agents Chemother. 2009; 53 (12): 5046-54. doi: 10.1128/AAC.00774-09.

37.- Nordmann P, Naas T, Poirel L. Global spread of carbapenemase producing Enterobacteriaceae. Emerg Infect Dis. 2011; 17 (10): 1791-8. doi: 10.3201/eid1710.110655

38.- Rada AM, Hernández Gómez C, Restrepo E Villegas MC. Distribución y caracterización molecular de betalactamasas en bacterias Gram negativas en Colombia, 2001-2016. Biomédica 2019; 39 (Supl 1): 199-220. Available from: https://doi.org/10.7705/biomedica.v39i3.4351.

39.- Higgins P G, Pérez-Llarena F J, Zander E, Fernández A, Bou G, Seifert H. OXA-235, a novel class $\mathrm{D} \beta$-lactamase involved in resistance to carbapenems in Acinetobacter baumannii. Antimicrob Agents Chemother. 2013; 57 (5): 2121-6. doi: 10.1128/AAC.02413-12.

40.- Kumarasamy K, Kalyanasundaram A. Emergence of Klebsiella pneumoniae isolate co-producing NDM-1 with KPC-2 from India J Antimicrob Chemother. 2012; 67 (1): 243-4. doi: $10.1093 / \mathrm{jac} / \mathrm{dkr} 431$.

41.- Correa A, Montealegre M C, Mojica M F, Maya J J, Rojas L J, De La Cadena E P, et al. First report of a Pseudomonas aeruginosa isolate coharboring KPC and VIM carbapenemases. Antimicrob Agents Chemother. 2012; 56 (10): 5422-3. doi: 10.1128/AAC.00695-12. 